\title{
On the number of connected and disconnected coverings over a manifold
}

\author{
Valery A. Liskovets * \\ Institute of Mathematics, National Academy of Sciences of Belarus \\ Minsk, 220072, Belarus \\ Alexander D. Mednykh ${ }^{\dagger}$ \\ Sobolev Institute of Mathematics, Novosibirsk State University \\ Novosibirsk, 630090, Russia
}

Received 5 February 2009, accepted 4 September 2009, published online 30 September 2009

\begin{abstract}
A general formula is obtained for the number of non-equivalent coverings (possibly disconnected) over a connected manifold with an arbitrary finitely generated fundamental group. Some illustrative examples are considered.
\end{abstract}

Keywords: Counting homomorphisms, non-equivalent coverings, disconnected coverings, fundamental group; Euler transform.

Math. Subj. Class.: 57M10, 20F34, 05A15, 14H30

\section{Introduction}

Let $p: \mathcal{U} \rightarrow \mathcal{M}$ be an arbitrary (possibly disconnected) covering over a connected manifold $\mathcal{M}$. Then every path $\gamma \in \mathcal{M}$ has a unique lifting $\widetilde{\gamma}$ starting at a given point of $p^{-1}(\gamma(0))$; so we obtain a well-defined mapping $L_{\gamma}: p^{-1}(\gamma(0)) \rightarrow p^{-1}(\gamma(1))$ by sending the starting point $\widetilde{\gamma}(0)$ of each lifting $\widetilde{\gamma}$ to its ending point $\widetilde{\gamma}(1)$. By the familiar monodromy theorem, $L_{\gamma}$ depends only on the homotopy class of $\gamma$. This means that the association $\gamma \mapsto L_{\gamma}$ gives rise to a homomorphism from $\pi_{1}\left(\mathcal{M}, x_{0}\right)$ to a permutation group acting on the fiber $F=p^{-1}\left(x_{0}\right)$. We note that this action of $\pi_{1}\left(\mathcal{M}, x_{0}\right)$ is transitive if and only if the covering

\footnotetext{
* Supported by the BRFFR (grant F07-293).

† Supported by the RFBR (grant 09-01-00255), APVV SK-RU-0007-07 and by Fondecyt (grants 7050189, 1060378).

E-mail addresses: liskov@im.bas-net.by (Valery A. Liskovets), mednykh@math.nsc.ru (Alexander D. Mednykh)
} 
manifold $\mathcal{U}$ is connected. More generally, the number of orbits of the action coincides with the number of connected components of the covering.

Two coverings $p_{1}: \mathcal{U}_{1} \rightarrow \mathcal{M}$ and $p_{2}: \mathcal{U}_{2} \rightarrow \mathcal{M}$ are equivalent if and only if the corresponding actions of $\pi_{1}\left(\mathcal{M}, x_{0}\right)$ on the fibers $F_{1}$ and $F_{2}$ over $x_{0}$ are isomorphic. This shows that $n$-sheeted covering spaces over $\mathcal{M}$ are classified by equivalence classes of homomorphisms $\rho: \pi_{1}\left(\mathcal{M}, x_{0}\right) \rightarrow \mathbf{S}_{n}$, where $\mathbf{S}_{n}$ is the symmetric group on $n$ symbols and the equivalence relation imposes an equivalence between $\rho$ and all its conjugates $h^{-1} \rho h$ by elements $h \in \mathbf{S}_{n}$.

All the above-mentioned results remain valid for any path-connected, locally pathconnected and semilocally simply connected topological space $\mathcal{M}$; see, for example [2, Ch.1.3: Th.1.38]. In particular, they are valid for finite graphs.

In the present paper we count the number of all non-equivalent coverings (connected or not) over a connected manifold with a finitely generated fundamental group. Then, by making use of the standard Euler transform (see Lemma 2.6 below) we relate the number of connected and disconnected coverings to get a new proof of the main result of [12]. This approach, which makes it possible to calculate the number of connected coverings through the number of disconnected ones, is, essentially, new. Earlier, for the special cases of the free group and the free product of cyclic groups, it has been effectively used in [4] and [15] to enumerate graph coverings and 'unsensed' maps on closed orientable surfaces, respectively.

Related general results for the enumeration of coverings were obtained by H. Tamanoi [18] and by T. Müller and J. Shareshian [13].

Several preceding papers contain formulae for counting non-equivalent connected and disconnected coverings. The numbers of non-equivalent connected and arbitrary coverings of a graph were determined in [7] and [4], respectively. Connected coverings of closed surface were counted in [10]. A general method to enumerate connected coverings over manifolds was given in [11, 12]. In more detail, the enumeration of graphs and manifold coverings (including disconnected ones) is considered in [6]. In [1], a relation between the numbers of connected and disconnected weighted ramified coverings over a torus is derived and used. Some enumerative applications of disconnected structures to chemistry are given in [14].

\section{Main results}

The following theorem has been proved by the second-named author [11, 12].

Theorem 2.1. Let $\mathcal{M}$ be a connected manifold with a finitely generated fundamental group $\Gamma$. Then the number $u_{n}$ of non-equivalent connected $n$-fold coverings over $\mathcal{M}$ is given by the formula

$$
u_{n}=\frac{1}{n} \sum_{\substack{\ell \mid n \\ \ell m=n}} \sum_{K<m}\left|\operatorname{Epi}\left(K, \mathbb{Z}_{\ell}\right)\right|,
$$

where the sum $\sum_{K<_{m} \Gamma}$ is taken over all subgroups $K$ of index $m$ in the group $\Gamma$, $\operatorname{Epi}\left(K, \mathbb{Z}_{\ell}\right)$ is the set of epimorphisms of the group $K$ onto a cyclic group $\mathbb{Z}_{\ell}$ of order $\ell$ and $|X|$ denotes the cardinality of a set $X$.

The aim of the present paper is to extend this theorem to coverings that are not necessarily connected. 
Theorem 2.2. Let $\mathcal{M}$ be a connected manifold with a finitely generated fundamental group $\Gamma$. Denote by $b_{n}$ the number of all non-equivalent (connected or disconnected) $n$-fold coverings over $\mathcal{M}$ and let $B(x)=1+b_{1} x+b_{2} x^{2}+\cdots$ be the ordinary generating function for these numbers. Then

$$
B(x)=\exp \left(\sum_{n=1}^{\infty} \sum_{\substack{\ell \mid n \\ \ell m=n}} \sum_{K<m}\left|\operatorname{Hom}\left(K, \mathbb{Z}_{\ell}\right)\right| \frac{x^{n}}{n}\right),
$$

where $\operatorname{Hom}\left(K, \mathbb{Z}_{\ell}\right)$ is the set of homomorphisms of the group $K$ into a cyclic group $\mathbb{Z}_{\ell}$.

Proof. From Burnside's lemma we obtain (reasoning similarly to [8]) the following result.

\section{Lemma 2.3.}

$$
b_{n}=\sum_{c_{1}+2 c_{2}+\cdots+n c_{n}=n} \prod_{i=1}^{n} \frac{\left|\operatorname{Hom}\left(\Gamma, \mathbb{Z}_{i} \operatorname{Wr} \mathbb{S}_{c_{i}}\right)\right|}{i^{c_{i}} c_{i} !},
$$

where $A \mathrm{Wr} B$ is the standard wreath product of permutation groups $A$ and $B$ and $\mathbb{Z}_{i}$ is considered as a regular cyclic permutation group of order $i$.

Since $n=c_{1}+2 c_{2}+\cdots+n c_{n}$, we have from this lemma,

$$
b_{n} x^{n}=\sum_{c_{1}+2 c_{2}+\cdots+n c_{n}=n} \prod_{i=1}^{n} \frac{\left|\operatorname{Hom}\left(\Gamma, \mathbb{Z}_{i} \operatorname{Wr} \mathbb{S}_{c_{i}}\right)\right|}{i^{c_{i}} c_{i} !} x^{i c_{i}} .
$$

Hence, $B(x)=\prod_{i=1}^{\infty} b_{i}(x)$, where

$$
b_{i}(x)=\sum_{c \geq 0} \frac{\left|\operatorname{Hom}\left(\Gamma, \mathbb{Z}_{i} \operatorname{Wr} \mathbb{S}_{c}\right)\right|}{i^{c} c !} x^{i c} .
$$

The next lemma follows directly from formula (5) of [13].

Lemma 2.4. For any $\ell \geq 1$,

$$
\sum_{m \geq 0} \frac{\left|\operatorname{Hom}\left(\Gamma, \mathbb{Z}_{\ell} \operatorname{Wr} \mathbb{S}_{m}\right)\right|}{m !} x^{m}=\exp \left(\sum_{m \geq 1} \ell^{m-1} \sum_{K<_{m} \Gamma}\left|\operatorname{Hom}\left(K, \mathbb{Z}_{\ell}\right)\right| \frac{x^{m}}{m}\right) .
$$

Now we return to the proof of Theorem 2.2. Taking (2.4) into account we have

$$
\begin{gathered}
b_{i}(x)=\sum_{c \geq 0} \frac{\left|\operatorname{Hom}\left(\Gamma, \mathbb{Z}_{i} \operatorname{Wr} \mathbb{S}_{c}\right)\right|}{i^{c} c !} x^{i c}=\sum_{c \geq 0} \frac{\left|\operatorname{Hom}\left(\Gamma, \mathbb{Z}_{i} \operatorname{Wr} \mathbb{S}_{c}\right)\right|}{c !}\left(\frac{x^{i}}{i}\right)^{c} \\
=\exp \left(\sum_{m \geq 1} \frac{i^{m-1} \sum_{K<_{m} \Gamma}\left|\operatorname{Hom}\left(K, \mathbb{Z}_{i}\right)\right|}{m}\left(\frac{x^{i}}{i}\right)^{m}\right) \\
=\exp \left(\sum_{m \geq 1} \sum_{K<_{m} \Gamma}\left|\operatorname{Hom}\left(K, \mathbb{Z}_{i}\right)\right| \frac{x^{i m}}{i m}\right) .
\end{gathered}
$$

Finally, we obtain

$$
B(x)=\prod_{i=1}^{\infty} b_{i}(x)=\exp \left(\sum_{i=1}^{\infty} \sum_{m \geq 1} \sum_{K<_{m} \Gamma}\left|\operatorname{Hom}\left(K, \mathbb{Z}_{i}\right)\right| \frac{x^{i m}}{i m}\right)
$$




$$
=\exp \left(\sum_{n=1}^{\infty} \sum_{\substack{\ell \mid n \\ \ell m=n}} \sum_{K<_{m} \Gamma}\left|\operatorname{Hom}\left(K, \mathbb{Z}_{\ell}\right)\right| \frac{x^{n}}{n}\right) .
$$

As a consequence of Theorem 2.2 we can give a new proof of Theorem 2.1. To that end we will make use of the following preliminary results [3]:

\section{Lemma 2.5.}

$$
\left|\operatorname{Epi}\left(K, \mathbb{Z}_{\ell}\right)\right|=\sum_{d \mid \ell} \mu\left(\frac{\ell}{d}\right)\left|\operatorname{Hom}\left(K, \mathbb{Z}_{\ell}\right)\right|,
$$

where $\mu(n)$ is the number-theoretic Möbius function.

In enumerative combinatorics, there is a simple general formula ${ }^{1}$ (see, e.g., [19, Th. 3.14.1]) called the Euler transform [17, p. 20] that relates the generating function of arbitrary, possibly disconnected, unlabeled objects of a certain kind with that of connected objects of the same kind (serving as their independent "building blocks"). In terms of coverings it is formulated as follows.

Lemma 2.6. Let $u_{i}$ and $B(x)$ be defined as in Theorems 2.1 and 2.2, respectively. Then the following identity is valid:

$$
B(x)=\prod_{i=1}^{\infty}\left(1-x^{i}\right)^{-u_{i}}
$$

Proof. Denote by $\mathfrak{S}$ the set of equivalence classes of all connected coverings of finite multiplicity over the manifold $\mathcal{M}$. Let $\mathfrak{D}=\mathbb{N S}$ be the set of all finite linear combinations of the type $m_{1} C_{1}+m_{2} C_{2}+\ldots+m_{k} C_{k}$, where $m_{1}, m_{2}, \ldots, m_{k} \in \mathbb{N}$ and $C_{1}, C_{2}, \ldots, C_{k} \in \mathfrak{S}$. We identify $\mathfrak{D}$ with the set of equivalence classes of all coverings of finite multiplicity over the manifold $\mathcal{M}$.

Define $\hat{\mu}: \mathfrak{S} \rightarrow \mathbb{N}$ to be the multiplicity of the covering. For any $d=m_{1} C_{1}+$ $m_{2} C_{2}+\ldots+m_{k} C_{k} \in \mathfrak{D}$, we set $\hat{\mu}(d)=m_{1} \cdot \hat{\mu}\left(C_{1}\right)+m_{2} \cdot \hat{\mu}\left(C_{2}\right)+\ldots+m_{k} \cdot \hat{\mu}\left(C_{k}\right)$. Then $u_{n}=|(c \in \mathfrak{S}: \hat{\mu}(c)=n)|$ and $b_{n}=|(d \in \mathfrak{D}: \hat{\mu}(d)=n)|$ are the numbers of equivalence classes of connected and all $n$-fold coverings, respectively.

Let $C_{1}^{i}, C_{2}^{i}, \ldots, C_{a_{i}}^{i} \in \mathfrak{S}$ be the list of equivalence classes of all $i$-fold connected coverings for any $i=1,2, \ldots$ Then any $n$-fold covering, up to equivalence, can be uniquely represented as $C=\sum_{i=1}^{n} \sum_{j=1}^{a_{i}} m_{j}^{i} C_{j}^{i}$ for some non-negative integers $m_{j}^{i}$ satisfying $\hat{\mu}(C)=n$.

Since $\hat{\mu}\left(C_{j}^{i}\right)=i$, the last condition is equivalent to $\sum_{i=1}^{n} \sum_{j=1}^{a_{i}} i \cdot m_{j}^{i}=n$. Hence, $b_{n}$ coincides with the number of non-negative solution of above equation. We set also $b_{0}=1$. Then we have

$$
b_{n} x^{n}=\sum_{\sum_{i=1}^{n} \sum_{j=1}^{u_{i}} i \cdot m_{j}^{i}=n} \prod_{i=1}^{n} \prod_{j=1}^{a_{i}} x^{i \cdot m_{j}^{i}}
$$

\footnotetext{
${ }^{1}$ In applications to unlabeled graphs it is also known as Riddell's formula.
} 
and, consequently,

$$
B(x)=b_{0}+b_{1} x+b_{2} x^{2}+\ldots=\prod_{i=1}^{\infty}\left(1+x^{i}+x^{2 i}+\ldots\right)^{u_{i}}=\prod_{i=1}^{\infty}\left(1-x^{i}\right)^{-u_{i}} .
$$

Now we are ready to prove Theorem 2.1 as a consequence of Theorem 2.2.

Proof. By Theorem 2.2 we have

$$
B(x)=\exp \left(\sum_{n=1}^{\infty} v_{n} \frac{x^{n}}{n}\right)
$$

where

$$
v_{n}=\sum_{\substack{\ell \mid n \\ \ell m=n}} \sum_{K<_{m} \Gamma}\left|\operatorname{Hom}\left(K, \mathbb{Z}_{\ell}\right)\right| .
$$

By Lemma 2.6 we also have

$$
B(x)=\prod_{i=1}^{\infty}\left(1-x^{i}\right)^{-u_{i}}
$$

Then, combining (2.5) and (2.6) and taking the logarithm we obtain

$$
\sum_{n=1}^{\infty} v_{n} \frac{x^{n}}{n}=-\sum_{i=1}^{\infty} u_{i} \log \left(1-x^{i}\right)
$$

After differentiating (2.7) and multiplying by $x$ we get

$$
\sum_{n=1}^{\infty} v_{n} x^{n}=\sum_{i=1}^{\infty} \frac{i u_{i} x^{i}}{1-x^{i}}
$$

Comparing the coefficients of the Taylor and Lambert series we have $v_{n}=\sum_{j \mid n} j u_{j}$. Now, by the Möbius inversion formula we obtain

$$
\begin{gathered}
n u_{n}=\sum_{j \mid n} \mu\left(\frac{n}{j}\right) v_{j}=\sum_{j \mid n} \mu\left(\frac{n}{j}\right) \sum_{m \mid j} \sum_{K<_{m} \Gamma}\left|\operatorname{Hom}\left(K, \mathbb{Z}_{\frac{j}{m}}\right)\right| \\
=\sum_{j \mid n} \sum_{m \mid j} \sum_{K<_{m} \Gamma} \mu\left(\frac{n}{j}\right)\left|\operatorname{Hom}\left(K, \mathbb{Z}_{\frac{j}{m}}\right)\right| \\
=\sum_{m \mid n} \sum_{m|j| n} \sum_{K<_{m} \Gamma} \mu\left(\frac{n}{j}\right)\left|\operatorname{Hom}\left(K, \mathbb{Z}_{\frac{j}{m}}\right)\right|=\sum_{m \mid n} \sum_{K<_{m} \Gamma} \sum_{m|j| n} \mu\left(\frac{n}{j}\right)\left|\operatorname{Hom}\left(K, \mathbb{Z}_{\frac{j}{m}}\right)\right| \\
=\sum_{\substack{\ell \mid n \\
\ell m=n}} \sum_{K<_{m} \Gamma} \sum_{d \mid \ell} \mu\left(\frac{\ell}{d}\right)\left|\operatorname{Hom}\left(K, \mathbb{Z}_{d}\right)\right|=\sum_{\substack{\ell \mid n \\
\ell m=n}} \sum_{K<_{m} \Gamma}\left|\operatorname{Epi}\left(K, \mathbb{Z}_{\ell}\right)\right|
\end{gathered}
$$

(the last equality follows from Lemma 2.5). This is formula (2.1). 


\section{Examples}

\subsection{The number of not necessarily connected coverings over a circle}

In this case, $\Gamma=\pi_{1}\left(S^{1}\right)=\mathbb{Z}$, an infinite cyclic group. The total number of $n$-fold coverings over the circle $S^{1}$ is given by the standard partition function $p(n)$, where

$$
p(0)+p(1) x+p(2) x^{2}+\cdots=\prod_{i=1}^{\infty}\left(1-x^{i}\right)^{-1}
$$

(so that, $p(0)=1, p(1)=1, p(2)=2, p(3)=3, p(4)=5, p(5)=7, p(6)=11, \ldots$ ). This is the well-known Euler equation. To derive it in the framework of our approach we note that the group $\mathbb{Z}$ has only one subgroup of index $n$. Hence, $u_{n}=1$ for every $n \geq 1$, and the result follows from Lemma 2.6.

\subsection{The number of coverings over a graph}

Let $G$ be a finite connected graph with Betti number $r=\beta(G)$. Then $\Gamma=\pi_{1}(G)=F_{r}$ is a free group of rank $r$. By $[7,8]$ we have $\left|\operatorname{Hom}\left(\Gamma, \mathbb{Z}_{i} \operatorname{Wr} \mathbb{S}_{c_{i}}\right)\right|=\left(i^{c_{i}} c_{i} !\right)^{r}$. Hence, by Lemma 2.3,

$$
b_{n}=\sum_{c_{1}+2 c_{2}+\cdots+n c_{n}=n} \prod_{i=1}^{n}\left(i^{c_{i}} c_{i} !\right)^{r-1} .
$$

This is a result by J. H. Kwak and Y. Lee [4]. It has been used to calculate the number of connected coverings over a graph $G$. The latter coincides with the number of conjugacy classes of subgroups of index $n$ in the free group $F_{r}$ obtained earlier in [7]. Thus, Theorem 2.2 gives an affirmative answer to the question posed by J. H. Kwak [5] as to whether (and how) it is possible to relate the enumerative results of papers [4] and [7].

\subsection{The number of all coverings over a closed orientable surface}

In this case we have

$$
B(x)=\exp \left(\sum_{n=1}^{\infty} \sum_{\substack{\ell \mid n \\ \ell m=n}} \ell^{(2 g-2) m+2} M_{\Phi_{g}}(m) \frac{x^{n}}{n}\right),
$$

where $\Phi_{g}$ is the fundamental group of a closed orientable surface of genus $g$ and $M_{\Phi_{g}}(n)$ is the number of subgroups of index $n$ in $\Phi_{g}$. Indeed, by the Riemann-Hurwitz formula, any subgroup $K$ of index $m$ in the group $\Phi_{g}$ is isomorphic to $\Phi_{g^{\prime}}$, where $\left(2 g^{\prime}-2\right)=(2 g-2) m$. Now, the first homology group $H_{1}\left(\Phi_{g^{\prime}}\right)=\Phi_{g^{\prime}} /\left[\Phi_{g^{\prime}}, \Phi_{g^{\prime}}\right]$ is equal to $\mathbb{Z}^{2 g^{\prime}}=\mathbb{Z}^{(2 g-2) m+2}$ and $\left|\operatorname{Hom}\left(K, \mathbb{Z}_{\ell}\right)\right|=\left|\operatorname{Hom}\left(H_{1}(K), \mathbb{Z}_{\ell}\right)\right|=\ell^{(2 g-2) m+2}$. Therefore,

$$
\sum_{K<_{m} \Gamma}\left|\operatorname{Hom}\left(K, \mathbb{Z}_{\ell}\right)\right|=\ell^{(2 g-2) m+2} M_{\Phi_{g}}(m) .
$$

Substituting this equality into the statement of Theorem 2.2 we obtain (3.2).

Recall [9] that $M_{\Phi_{g}}(m)$ can be computed by the following linear recursion formula:

$$
M_{\Phi_{g}}(m)=m \beta_{m}-\sum_{j=1}^{m-1} \beta_{m-j} M_{\Phi_{g}}(j), \quad M_{\Phi_{g}}(1)=1
$$


where

$$
\beta_{k}=\sum_{\chi \in D_{k}}\left(\frac{k !}{f \chi}\right)^{2 g-2}
$$

$D_{k}$ is the set of all irreducible representations of the symmetric group $\mathbf{S}_{k}$ and $f^{\chi}$ is the degree of a representation $\chi$.

Instead of (3.2), by the Euler transform (Lemma 2.6), we have immediately,

$$
B(x)=\prod_{i=1}^{\infty}\left(1-x^{i}\right)^{-u_{i}}
$$

where $u_{n}=N_{\Phi_{g}}(n)$ denotes the number of connected $n$-fold coverings of a closed orientable surface of genus $g$, which is equal to the number of conjugacy classes of subgroups of index $n$ in $\Phi_{g}$ found in [9, 10]. Equating the right-hand sides of (3.2) and (3.5), we obtain the following equation for $N_{\Phi_{g}}(n)$ in terms of generating functions:

$$
\prod_{i=1}^{\infty}\left(1-x^{i}\right)^{-N_{\Phi_{g}}(i)}=\exp \left(\sum_{n=1}^{\infty} \sum_{\substack{\ell \mid n \\ \ell m=n}} \ell^{(2 g-2) m+2} M_{\Phi_{g}}(m) \frac{x^{n}}{n}\right), \quad g \geq 0
$$

which is equivalent to the explicit formula obtained in [9].

Let $b_{n}=D C(n, \nu)$ be the total number of all non-equivalent $n$-fold coverings (connected or disconnected) over a closed orientable surface of genus $g$, where $\nu=2 g-2$ is its characteristic. Then by formula (3.2) we calculated for $n=1, \ldots, 7$ :

$$
\begin{aligned}
D C(1, \nu)= & 1 \\
D C(2, \nu)= & 4 \cdot 2^{\nu} \\
D C(3, \nu)= & 2 \cdot 6^{\nu}+4 \cdot 3^{\nu}+2 \cdot 2^{\nu} \\
D C(4, \nu)= & 2 \cdot 24^{\nu}+12^{\nu}+6 \cdot 8^{\nu}+9 \cdot 4^{\nu}+3 \cdot 3^{\nu} \\
D C(5, \nu)= & 2 \cdot 120^{\nu}+2 \cdot 30^{\nu}+2 \cdot 24^{\nu}+20^{\nu}+4 \cdot 12^{\nu}+4 \cdot 8^{\nu}+14 \cdot 6^{\nu} \\
& +5 \cdot 5^{\nu}+5 \cdot 4^{\nu} \\
D C(6, \nu)= & 2 \cdot 720^{\nu}+4 \cdot 144^{\nu}+2 \cdot 80^{\nu}+2 \cdot 72^{\nu}+8 \cdot 48^{\nu}+45^{\nu}+4 \cdot 24^{\nu} \\
& +12 \cdot 18^{\nu}+16 \cdot 16^{\nu}+6 \cdot 9^{\nu}+18 \cdot 8^{\nu}+12 \cdot 6^{\nu}+5 \cdot 5^{\nu} \\
D C(7, \nu)= & 2 \cdot 5040^{\nu}+2 \cdot 840^{\nu}+4 \cdot 360^{\nu}+2 \cdot 336^{\nu}+252^{\nu}+6 \cdot 240^{\nu}+2 \cdot 144^{\nu} \\
& +6 \cdot 72^{\nu}+4 \cdot 60^{\nu}+16 \cdot 48^{\nu}+2 \cdot 40^{\nu}+3 \cdot 36^{\nu}+34 \cdot 24^{\nu}+6 \cdot 18^{\nu} \\
& +4 \cdot 16^{\nu}+32 \cdot 12^{\nu}+20 \cdot 10^{\nu}+3 \cdot 9^{\nu}+8 \cdot 8^{\nu}+7 \cdot 7^{\nu}+6 \cdot 6^{\nu} .
\end{aligned}
$$

Table 1 contains initial numerical values of the function $D C(n, \nu), \nu \geq-2$. For completeness we included values for odd $\nu$ as well, in particular, fractional values for $\nu=-1$. The latter have a simple combinatorial meaning: $D C(n,-1)=r^{2}(n) / n$ !, where $r(n)$ is the sum of the degrees of the irreducible representations of the symmetric group $\mathbf{S}_{n}$ (cf. formula (3.4) $)^{2}$.

\footnotetext{
${ }^{2}$ This is the sequence A000085 [16]. The generating function for $r^{2}(n) / n !$ is $\exp (x /(1-x)) / \sqrt{1-x^{2}}$, and the values of $r^{2}(n)$ form the sequence A111883.
} 


\begin{tabular}{|r|r||r|r|r|r|r|r|r|}
\hline$\nu$ & $g$ & 1 & 2 & 3 & 4 & 5 & 6 & 7 \\
\hline-2 & 0 & 1 & 1 & 1 & 1 & 1 & 1 & 1 \\
-1 & - & 1 & 2 & $16 / 3 !$ & $100 / 4 !$ & $676 / 5 !$ & $5776 / 6 !$ & $53824 / 7 !$ \\
0 & 1 & 1 & 4 & 8 & 21 & 39 & 92 & 170 \\
1 & - & 1 & 8 & 28 & 153 & 577 & 3612 & 19228 \\
2 & 2 & 1 & 16 & 116 & 1851 & 33693 & 1175852 & 53529098 \\
3 & - & 1 & 32 & 556 & 33105 & 3558577 & 761393916 & 257606692684 \\
4 & 3 & 1 & 64 & 2948 & 711411 & 417285429 & 539383049612 & \\
5 & - & 1 & 128 & 16588 & 16380633 & 49835381257 & 387243914607612 & \\
6 & 4 & 1 & 256 & 96356 & 386803851 & 5973885950253 & & \\
\hline
\end{tabular}

Table 1: Values of $D C(n, \nu)$ for $\nu \leq 6$ and $n \leq 7$.

\subsection{The number of coverings over a torus}

In conclusion we address the particular case $\nu=0$. By [10] we have $M_{\Phi_{1}}(n)=\sigma(n)$, where $\sigma(n)=\sum_{d \mid n} d$ is the sum of positive divisors of $n$. Setting $g=1$ in formula (3.2) we obtain

$$
B(x)=\exp \left(\sum_{n=1}^{\infty} \sum_{\substack{\ell \mid n \\ \ell m=n}} \ell^{2} \sigma(m) \frac{x^{n}}{n}\right) .
$$

Again, instead of (3.7), by the Euler transform we have

$$
B(x)=\prod_{i=1}^{\infty}\left(1-x^{i}\right)^{-\sigma(i)} .
$$

Accordingly, $b_{n}=D C(n, 0), n \geq 1$, is the sequence A061256 [16], which starts with $1,4,8,21,39,92,170,360,667,1316,2393,4541,8100,14824,26071,46422$.

\section{Acknowledgements}

We thank the anonymous referees for reading our paper carefully and giving many helpful suggestions concerning its text.

\section{References}

[1] A. Eskin and A. Okounkov, Asymptotics of numbers of branched coverings of a torus and volumes of moduli spaces of holomorphic differentials, Invent. Math. 145 (2001), 59-103.

[2] A. Hatcher, Algebraic Topology, Cambridge University Press, Cambridge, 2002.

[3] G. A. Jones, Enumeration of homomorphisms and surface-coverings, Quart. J. Math. Oxford (2) 46 (1995), 485-507.

[4] J. H. Kwak and J. Lee, Enumeration of connected graph coverings, J. Graph Theory 23 (1996), 105-109.

[5] J. H. Kwak and J. Lee, Enumeration of graph coverings, surface branched coverings and related group theory, in: S. Hong, J. H. Kwak et al. (eds.), Combinatorial Computational Mathematics: Present and Future, Word Scientific, Singapore, 2001, 97-161.

[6] J. H. Kwak and A. D. Mednykh, Enumeration of Graphs and Manifold Coverings, $\mathrm{Com}^{2} \mathrm{Mac}$ Lect. Notes, No. 19, Pohang: Pohang Univ. of Science and Technology, 2007. 
[7] V. A. Liskovets, On the enumeration of subgroups of a free group, Dokl. Akad. Nauk BSSR 15 (1971), 6-9.

[8] V. A. Liskovets, Reductive enumeration under mutually orthogonal group actions, Acta Applic. Math. 52 (1998), 91-120.

[9] A. D. Mednykh, On the Hurwitz problem on the number of nonequivalent coverings over a compact Riemann surface, Sib. Math. J. 23 (1983), 415-420; translation from Sib. Mat. Zh. 23 (1982), 155-160.

[10] A. D. Mednykh, On the number of subgroups in the fundamental group of a closed surface, Commun. Algebra 16 (1988), 2137-2148.

[11] A. D. Mednykh, A new method for counting coverings over manifold with finitely generated fundamental group, Doklady Mathematics 74 (2006), 498-502; translation from Dokl. Akad. Nauk 409 (2006), 158-162.

[12] A. D. Mednykh, Counting conjugacy classes of subgroups in a finitely generated group, $J$. Algebra 320 (2008), 2209-2217.

[13] T. W. Müller and J. Shareshian, Enumerating representations in finite wreath products, II: Explicit formulae, Adv. Math. 171 (2002), 276-331.

[14] M. Petkovšek and T. Pisanski, Counting disconnected structures: chemical trees, fullerenes, I-graphs, and others, Croatica Chemica Acta 78 (2005), 563-567.

[15] R. Robinson, Counting unrooted maps of all genera, Talk at the Twentieth Clemson MiniConference on Discrete Math. and Related Fields, Oct. 2005, http://www.cs.clemson. edu/ goddard/MINI/2005/Robinson.pdf.

[16] N. J. A. Sloane, On-Line Encyclopedia of Integer Sequences (2009), http://www.research.att.com/ njas/sequences/.

[17] N. J. A. Sloane and S. Plouffe, The Encyclopedia of Integer Sequences, Academic Press, San Diego, CA, 1995.

[18] H. Tamanoi, Generalized orbifold Euler characteristics of symmetric orbifolds and covering spaces, Algebr. Geom. Topol. 3 (2003), 791-856 (electronic).

[19] H. S. Wilf, generatingfunctionology, Second edition, Academic Press, Boston, MA, 1994. 